\title{
Editorial
}

\section{Metasurfaces - from science to applications}

https://doi.org/10.1515/nanoph-2018-0064

Metasurfaces are artificial photonic structures that allow exceptional control of the optical wavefront over deeply subwavelength thicknesses, realizing several optical functionalities of interest, including beam steering, focusing, and holography, in planarized devices that are extremely thin. In order to enable this unusual response, it is imperative to strongly enhance the interaction between light and matter. Multiple techniques have been explored in this context, which range from dielectric arrayed resonators, to plasmonic antennas or waveguide-like nanostructures.

This special issue includes contributions of leading experts in the field of metasurfaces, surveying the latest approaches to manipulate light in extreme ways over a surface, reviewing, and detailing both metasurface-based scientific advances and technological opportunities in this field. This collection of articles is dedicated to a pioneering scientist - Dr. Federico Capasso - one of the leading experts in this field. We open the issue with a personal review article from Dr. Capasso himself on "The future and promise of flat optics: a personal perspective".

A question still being debated and actively explored in the field is the choice of materials for metasurfaces, because their resulting properties determine the response and thus the application functionality. The review article "Materials platforms, metasurface, plasmonics, dielectric metasurface" by Choudhury et al. discusses a wide variety of metasurface materials to include dielectric, metallic, meta-oxides, 2-dimensional materials, and semiconductors.

\section{Dielectric metasurfaces}

The article "Metasurfaces and their applications" by Li et al. provides a general overview of metasurface research, reviewing a variety of physical concepts that enable various functionalities, including beam shaping, cloaking, polarization and phase control, amplitude or phase modulation, and imaging.

A goal in metasurface technology is to enable efficient harmonic generation using nonlinear phenomena. The paper "Controlling the phase of optical nonlinearity with plasmonic metasurfaces" by Chen et al. shows how tailoring the local polarizability allows controlling both the amplitude and phase of nonlinear phenomena by using the rotational symmetry of the meta-atom. The key here is that the inversion of the incoming light being circularly polarized is accompanied by a geometric phase, called the Pancharatnam-Berry phase. This leads to an antenna-orientation controlled phase that does not depend on the specific antenna design or wavelength of light. Such phase-control can be used in nonlinear holography, vortex beam generation for OAM or two-dimensional flat optical lenses.

Many metasurface applications and demonstrations aim to control a free-space light beam reflecting off, or transmitting through the metasurface. The contribution "Molding light with metasurfaces: from far-field to near-field interactions" by Gangaraj and Monticone, instead, discusses how metasurfaces enable near-field effects to control the generation and propagation of surface waves. For instance, anisotropic and hyperbolic metasurfaces show some of the most exciting and extreme examples of anomalous surface-wave propagation on planarized artificial structures, with important implications for light focusing, confinement, and subwavelength imaging. The authors also connect these ideas with the emerging area of two-dimensional materials, and discuss how to implement hyperbolic metasurfaces with graphene and black phosphorus.

The contribution "A review of dielectric optical metasurfaces for wavefront control" by Kamali et al. provides an overview of recent developments of metasurfaces made of dielectric structures. In particular, they show how to achieve steep phase gradients with high efficiency, simultaneous polarization and phase control, control of the chromatic dispersion, or control of the angular response. The low-optical absorption of dielectric-based metasurfaces enables applications in imaging, conformal optics, tunable devices, and optical systems.

The two articles "Bianisotropic metasurfaces: physics and applications" by Asadchy et al. and "Design, concepts and applications of electromagnetic metasurfaces" by Achouri and Caloz discuss, amongst others, bianisotropy in metasurfaces. These are specific metasurfaces providing strong magnetoelectric coupling at the surface, which enables 
unique opportunities for wavefront control. Engineering this response is required for many key field transformations, such as efficient anomalous refraction, asymmetric reflection, polarization transformation, isolation, and more. The second contribution discusses an analytical approach to describe these surfaces using a generalized sheet transition condition. Using this approach, the authors discuss five recent metasurface concepts and applications, which cover the topics of birefringent transformations, bianisotropic refraction, light emission enhancement, remote spatial processing and nonlinear second-harmonic generation.

\section{ENZ metasurfaces}

Another contribution to this special issue explores the interesting field of vanishing index of refraction in metasurfaces. In zero index materials, the wavefront of an optical beam is effectively flattened, with unique properties when implemented in metasurface-like structures, nanophotonic elements, or, like in this contribution, used to create "Nanophotonics reconfigurable epsilon-near-zero metasurfaces via photonic doping" by Liberal and Engheta. Here, the transmission spectrum of the metasurface is characterized by a Fano-like resonance, where the metasurface behavior changes from perfect-magnetic-conductor to epsilon-and-mu-near-zero material responses. The abrupt variation between these two extreme materials responses opens potential applications for dynamic metasurfaces. Applications of this work therefore point to new strategies for dynamic wavefront engineering.

\section{Plasmonic metasurfaces}

Several contributions to this special issue are focused on plasmonic implementations of metasurfacces, such as the article "A review of gap-surface plasmon metasurfaces: fundamentals and applications" by Ding et al. Such gap-based metasurfaces consist of a subwavelength thin dielectric spacer sandwiched between an optically thick metal film and arrays of metal subwavelength elements arranged in a strictly or quasi-periodic fashion. With a single lithograph step, these metasurfaces feature ease of fabrication, yet allow phase, amplitude and polarization control of the reflected light. This contribution discusses the generalized Snell's law of refraction and explains the concept of the Pancharatnam-Berry phase.

The contribution "Dimerized high contrast gratings" by Yu et al. explores the case of high-contrast-gratings to control light on a surface. This contribution discusses symmetry-broken HCGs called dimerized high-contrast-gratings, which have a period-doubling perturbation applied. Such structures allow for compact devices due to large in-plane scattering with control of long lifetime due to the perturbation.

By employing the concepts of holographic multiplexing, multiple information channels, such as wavelength, polarization state, spatial position, and nonlinear frequency conversion, can be enabled in a metasurface platform, as discussed in "Metasurfaces holography: from fundamentals to applications" by Huang et al. Switchable metasurface holography by integration of functional materials stimulates a gradual transition from passive to active elements. Importantly, the holography principle has become a universal and simple approach to solving inverse engineering problems for electromagnetic waves.

\section{Acoustic, microwave, and non-Hermitian metasurfaces}

In addition to electromagnetic waves being controlled by metasurfaces, similar concepts apply to other types of waves as well. The article "Wavefront manipulation by acoustic metasurfaces: from physics and applications" by Liang et al., for instance, discusses acoustic metasurfaces which, in general, allow for similarly unique functionalities for sound, such as lensing, phase, and amplitude control, etc. Challenges in the field of acoustic metamaterials consist in finding mechanisms and designs with low frequency dispersion, beyond utilizing resonances.

Huygens' metasurfaces comprise a thin layer of orthogonal electric and magnetic dipoles that form an array of Huygens' sources, which radiate mostly in the forward direction and can be used to manipulate an incident electromagnetic wave at will, as discussed in "Huygens' metasurfaces from microwaves to optics: a review” by Chen et al. Examples 
of such passive manipulations include reflectionless refraction, perfect anomalous reflection, arbitrary antenna beam forming and chiral polarization control, while active Huygens' metasurfaces include cloaking and subwavelength spot formation in a cavity environment.

Contrary to conventional phase gradient metasurfaces where each meta-atom responds individually, the article "Non-conventional metasurfaces: from non-Hermitian coupling, quantum interactions, to skin cloak" by Ren et al. focuses on developing metasurfaces where neighboring meta-atoms are strongly coupled. By engineering anti-Hermitian coupling between the meta-atoms, new degrees of freedom are introduced, and novel functionalities can be achieved, such as ultrathin metasurface cloak and strong photonic spin-Hall effects.

\section{Outlook and potential challenges}

The development and study of optical metasurfaces have been a rapidly growing field of research in the past few years, because of their capabilities to mimic the functionality of conventional diffractive optical elements with higher efficiencies and resolutions, and more importantly for their advantages in providing new functionalities not achievable with conventional diffractive optics. Their subwavelength thickness, planar form factor, compatibility with conventional micro/nano-fabrication techniques, potentially low-cost batch fabrication, ability to replace a system of multiple bulky conventional elements with a miniature element, new capabilities to control different degrees of freedom of light, and prospects for a paradigm change in how optical systems are designed, make them very promising for the realization of the next generation of compact high-performance optical systems.

However, despite such advancements, several challenges still remain unsolved, both from a fundamental but also application point-of-view. For example, an important theoretical issue is the number of available degrees of freedom that exist in a single surface or a specific volume. This would determine the number of functionalities that can be encoded in such a device with negligible performance degradation. Another area that requires significant advancements is the modeling and design of non-periodic metasurfaces; while many designs utilize slowly varying metasurfaces, new design explorations in terms of abrupt-changing and non-periodic are of significant interest. In addition to high-efficiency high-NA devices, such methods could also allow for the design and analysis of novel metasurfaces that are not bound by the assumption of locality. The realization of achromatic and dispersion-engineered metasurfaces with practical sizes and moderate-to-high numerical apertures still demand further development.

In addition to fundamental challenges, unresolved practical issues limit the realization of high-volume low-cost metasurface devices for real-life applications. For example, the absence of a low-loss high-index material for visible light still shows an efficiency discrepancy between infrared (IR) or near infrared (NIR) operating metasurfaces and those at visible frequencies. Lastly, the manufacturing processes typically used for lab-type demonstrations do typically not go beyond electron beam lithography methods, and reliability tests using commercially used deep-UV lithography systems may bring the promise the of this young technology to the next market level. Finally, the achievable tunability range of reconfigurable metasurfaces still lags behind what is possible to achieve with commercial spatial light modulators, such as those based on liquid-crystals.

\section{Andrea Alu}

UT Austin, and SUNY in New York

\section{Vladimir Shalaev}

Purdue University

Marko Loncar

Harvard University

(Guest Editors)

\section{Volker J. Sorger}

George Washington University

(Editor-in-Chief) 